Vier-Tage-Woche

\section{Kein Wundermittel}

Die kurzfristigen negativen Auswirkungen der Corona-Krise auf den deutschen Arbeitsmarkt konnten dank Milliardenausgaben für Kurzarbeitergeld und Konjunkturpakete abgefedert werden: Die Zahl der Arbeitslosen erhöhte sich zwar bis August 2020 um gut eine halbe Million auf knapp 3 Mio., sie nähert sich aber keineswegs früheren Negativrekorden von 5 Mio. Arbeitslosen. Auch wenn der Bezug von Kurzarbeitergeld auf zwei Jahre mit Ersatzleistungen von bis zu $87 \%$ des letzten Nettoeinkommens sowie die Aussetzung der Insolvenzregelung bis Ende 2020 vom Kabinett beschlossen wurden, wird ein massiver Strukturwandel mit dem massenhaften Verlust von Industriejobs befürchtet. Vor diesem Hintergrund hat sich der Chef der IG Metall, Jörg Hofmann, für die Einführung der Vier-TageWoche in der gesamten Metall- und Elektroindustrie ausgesprochen - mit einem gewissen Lohnausgleich für die Beschäftigten. Seiner Ansicht nach ist eine Arbeitszeitverkürzung auch im Interesse der Arbeitgeber, um Kosten für einen Sozialplan bei Entlassungen einzusparen.

Bereits 2018 stand eine Arbeitszeitverkürzung auf 28 Stunden im Zentrum der Tarifverhandlungen. Der Kompromiss der Tarifvertragsparteien sah erstmals die Möglichkeit einer „verkürzten Vollzeit“ von 28 Stunden für maximal zwei Jahre vor, wenn Arbeitnehmer z.B. eine Familienzeit, eine Pflegezeit für Angehörige oder Zeit für eine Weiterbildung wünschten. Doch auch mehr Arbeitsstunden konnten befristet auf Wunsch des Arbeitgebers vereinbart werden. Damit war der tarifvertragliche Rahmen für eine „atmende" Arbeitszeit gesetzt. Ist eine Vier-Tage-Woche jedoch auch eine Lösung für den industriellen Strukturwandel, der durch den krisenbedingten Digitalisierungsschub des Jahres 2020 beschleunigt wird? Richtig ist: Als Folge der industriellen Revolution (erstes Maschinenzeitalter) stieg die Arbeitsproduktivität, sodass im Durchschnitt kürzere Arbeitszeiten bei höheren Stundenlöhnen in allen Industrieländern möglich wurden. Dementsprechend könnte als Folge der derzeitigen „Digitalisierungsrevolution“ (zweites Maschinenzeitalter), die seit Jahren im Durchschnitt stagnierende Arbeitsproduktivität deutlich zunehmen, sodass zukünftig auch niedrigere durchschnittliche Jahresarbeitszeiten bei höheren Löhnen möglich würden.

Jedoch sind solche langfristigen Entwicklungen über viele Jahrzehnte mit enorm schmerzhaften Anpassungsprozes-

(C) Der/die Autor(en) 2020. Open Access: Dieser Artikel wird unter der Creative Commons Namensnennung 4.0 International Lizenz (https:// creativecommons.org/licenses/by/4.0/deed.de) veröffentlicht.

Open Access wird durch die ZBW - Leibniz-Informationszentrum Wirtschaft gefördert. sen auf dem Arbeitsmarkt verbunden. So hat es während der industriellen Revolution viele Gewinner und Verlierer bei Arbeitgebern und Arbeitnehmern gegeben. Das ist auch zukünftig zu erwarten, wenn man alle empirischen Studien zu den Beschäftigungswirkungen der Digitalisierung zusammenfasst: Die meisten Studien kommen zu dem Ergebnis, dass die Nettobeschäftigung zwar konstant bleibt oder sogar zunimmt, jedoch viele Tätigkeiten und Stellen dauerhaft verschwinden. Dagegen werden neu geschaffene Stellen dank Fachkräftemangels nur sehr mühsam besetzt werden können.

Falsch ist jedoch der gedankliche Ansatz der IG Metall. Wenn die Arbeitszeitverkürzung bei einem gewissen Lohnausgleich dazu dienen soll den Strukturwandel zu erleichtern, dann steckt dahinter die Vorstellung eines festen „Arbeitskuchens": Das Volumen der Arbeitsstunden ist vorgegeben und muss verteilt werden. Wenn die Zahl der von den Unternehmen benötigten Arbeitsstunden krisenbedingt sinkt, dann muss die verbliebene Arbeit auf möglichst viele Köpfe verteilt werden, um Entlassungen zu vermeiden. Die logische Konsequenz aus Sicht der „Kuchentheoretiker“: Die Arbeitszeit pro Kopf muss sinken. Diese auf den ersten Blick plausible Logik ist theoretisch und empirisch längst widerlegt. Tatsächlich wirkt eine Arbeitszeitverkürzung bei weitgehendem Lohnausgleich als "Produktivitätspeitsche“ - die Arbeitnehmer werden von den Arbeitgebern unter Stress gesetzt, um in der verbliebenen Arbeitszeit mehr zu leisten, damit die erhöhten Stundenlöhne erwirtschaftet werden können. Die Vier-Tage-Woche ist deshalb kein Wundermittel gegen den beschleunigten Strukturwandel in der Industrie. Dagegen ist eine verringerte Jahresarbeitszeit bei höheren Löhnen langfristig vorstellbar - als Folge digitalisierungsbedingter Arbeitsproduktivitätszuwächse. Diese Zukunftsvision ist realistisch, wenn Deutschland bei der Digitalisierung nicht nur beim Homeoffice und der Industrie 4.0 ernst machen würde.

Kurzfristig werden durch die Initiative der IG Metall Illusionen genährt und falsche Erwartungen geweckt. Die Gewerkschaften sollten stattdessen die Themen VertrauensArbeitsort und Vertrauens-Ruhezeit auf ihre Agenda setzen. Denn wenn im Homeoffice mehr gearbeitet wird, wie aktuelle Studien vermuten lassen, und die zunehmende Verschmelzung von Arbeits- und Freizeit zu Dauerstress führt, dann geraten Errungenschaften gewerkschaftlicher Arbeit in Gefahr. Auch sollten die Tarifparteien den Gesetzgeber dazu drängen, das Arbeitszeitgesetz des letzten Jahrtausends zu aktualisieren, um mehr Arbeitszeitflexibilität zu ermöglichen.

Alexander Spermann

FOM Hochschule für Erwerbstätige Köln; Universität Freiburg spermann@alexander-spermann.de 